\title{
3 Research Square

\section{Impact and Clinical Implications of the Cognitive Function and Nutritional Assessment at Admission on Hospital Outcomes and Mortality in Acutely III Geriatric Patients}

\author{
Hiroshi Yamamoto ( $\nabla$ yamagen1006@gmail.com ) \\ Yamamoto Memorial Hospital https://orcid.org/0000-0001-9085-1383 \\ Kenichi Ogawa \\ Yamamoto Memonial Hospital \\ Toshifumi Morooka \\ Yamamoto Memorial Hospital \\ Yasushi Hisamatsu \\ Yamamoto Memorial Hospital \\ Tatsuya Ishitake \\ Kurume Daigaku
}

\section{Research article}

Keywords: Keywords: Cognitive impairment, Malnutrition, Revised Simplified Short-Term Memory Recall test (STMT-R), Short-Form Mini-Nutritional Assessment (MNA-SF) Topic: Acutely ill geriatric patients, Length of hospitalization, Hospital mortality

Posted Date: January 24th, 2020

DOI: https://doi.org/10.21203/rs.2.21750/v1

License: (c) (i) This work is licensed under a Creative Commons Attribution 4.0 International License. Read Full License 


\section{Abstract}

Background The nutritional and mental status of older patients are closely related and may serve as an indicator of hospital outcomes and early nutrition interventions.

Objective We aim to appraise the impact on mortality and hospital outcomes by the revised simplified short-term memory recall test (STMT-R) and the short-form mini-nutritional assessment (MNA-SF) simultaneous at admission.

Methods The subjects were 727 acute inpatients with $\geq 50$ years of age from December 2014 to September 2015. Following the collection of clinical data (base illness, length of stay, etc.), survival was subsequently measured for $4-5$ years until September 2019. An STMT-R score of $\leq 4$ and MNA-SF score $\leq 7$ were considered to indicate cognitive dysfunction and malnourishment respectively. Cox's proportional hazards regression models were used to evaluate the hazard of death according to the severity of dementia and the nutrition status, with adjustment for potential covariates. Survival was estimated using the Kaplan-Meier method.

Results The mean age of the study population was 78.9 years old, and $52.7 \%$ of the patients were female. 118 patients could not complete the cognitive test (Incomplete Testing Group [ITG]). 385 and 224 patients were classified into the cognitive dysfunction (CDG) and non-cognitive dysfunction groups (NCDG), respectively. 370, 299 and 58 patients were classified into the "malnourished" (MNG), "at risk of malnutrition" (ARNG) and "well-nourished" (WNG) groups, respectively. A poor cognitive function and poor nutritional status at admission were associated with worse clinical indicators. 276 patients (38.0\%) died during the 4 to 5 years of follow-up. The risks of mortality in the ITG, CDG and MNG was 3.88 (hazard ratio; $95 \%$ confidence interval: $2.57-5.98), 1.84$ (1.28-2.72) and 4.22 (1.76-13.9), respectively. In addition, the cumulative survival rate was $23.6 \%$ for the combination of advanced dementia and malnutrition, but $51.0 \%$ for moderate dementia and malnutrition. Even undernourishment may improve the prognosis of patients with mild to moderate cognitive impairment.

Conclusion The early simultaneous measurements of the cognitive function and nutritional status is expected to be useful for predicting the the hospital prognosis, mortality and the effects of early comprehensive nutritional intervention in acute elderly patients with cognitive decline.

\section{Introduction}

Cognitive impairment is one of the geriatric syndromes, including mild cognitive impairment, delirium and all stages of dementia, but is often difficult to detect in modern acute care [1,2]. Prompt recognition of cognitive dysfunction with sensitive screening is essential to improve emergency care and prognosis in the elderly [1]. Moreover, cognitive impairment and dementia can be important predictors of disability, dependence and death among older adults [3,4]. Malnutrition is also associated with a multitude of geriatric syndromes, including cognitive dysfunction, suggesting a relationship between the nutrition status and cognitive function [4,5]. Several studies have shown that malnutrition is associated with 
cognitive decline in older adults [6]. Globally, the decline in cognitive function associated with malnutrition is expected to further burden already-strained health care system due to the continued expansion of the aging population and the extension of life expectancy [4].

In Japan, the birth rate has been below the replacement level in recent decades. In other words, while the average life expectancy is getting longer, Japanese society is aging rapidly [7]. As a result, an increase in the population of dementia is also expected. In fact, the current official data shows that there are 4.62 million dementia patients, representing about $15 \%$ of the elderly population in Japan $[8,9]$. Moreover, in $2030,8.3$ million people, equivalent to about $23.2 \%$ of the elderly population in Japan, will become dementia patients, and it is expected that the Japanese healthcare system will be further burdened [9].

People with dementia are hospitalized frequently, but little is known about the health profile and prognosis of patients with dementia who access care in acute wards [10]. Multiple observational studies have shown that emergency physicians and nurses often do not recognize cognitive dysfunction [11]. More generally, physicians often fail to recognize cognitive impairment in elderly patients [1]. Indeed, it was reported that over $80 \%$ of emergency department (ED) patients with cognitive dysfunction lacked a diagnosis of dementia in the United States [12].

In contrast, the prevalence of malnutrition among hospitalized older adults and its consequences are well established [13]. Cognitive decline is cited as a frequent and common finding among hospitalized patients who are malnourished [14]. Several studies have found that more than $80 \%$ of newly admitted geriatric patients present with malnutrition or are at risk of malnutrition $[15,16]$. Continued before and after hospitalization, many conditions such as underlying diseases, co-morbidities, inflammatory conditions, and infections increase patient's energy consumption while reducing normal intake [16]. Therefore, all patients should undergo an assessment of their nutritional status at all stages of hospitalization [16].

The prompt recognition of cognitive dysfunction is also essential for high-quality geriatric emergency care in order to prevent unnecessary errors and complications [1]. Therefore, the use of a simple and sensitive cognitive screening system early in a hospital admission could ensure appropriate care for cases of cognitive decline, thereby shortening the duration of hospitalization, reducing medical expenses and decreasing mortality [17]. Multiple screening tests for the cognitive function have been proposed [17]. The Mini-Mental State Examination (MMSE) was developed in 1975 and has since become a widely used and accepted method for recognizing cognitive impairment [17]. However, the test itself takes roughly 6 minutes on average (3.5-14 minutes) [12], which can constitute a substantial burden for clinicians examining acutely ill geriatric patients [17]. Therefore, we adopted the Simplified Short-Term Memory Recall Test (STMT) [18] at our hospital, and for several years, we have been investigating the detection of cognitive impairment in our ED patients using this approach. In addition, given the difficulty for some groups of elderly patients to answer the STMT, we developed a slightly revised and modified version, called the STMT-R [17]. 
Evaluations of the nutritional status of the elderly, especially for patients with dementia, can be done in a variety of ways, depending on malnutrition indicators, anthropometric measurements, and oral intake assessment [19]. The Mini Nutrition Assessment (MNA) is the best validated and most widely utilized screening test for malnutrition among elderly populations because it was developed to assess the risk of malnutrition in the elderly and to identify those who are malnourished [20]. We adopted the MNA-Short Form (MNA-SF), which is a revised version of the MNA, at our hospital, and similarly to our efforts with the STMT/STMT-R, for several years, we have been investigating the detection of malnutrition in our acute care patients using this approach. This evaluation consists of six items, including anthropometric, nutritional and clinical evaluations as well as the self-perception of health [20]. The MNA-SF is used by trained health professionals and nutritionists for both screening and evaluating the nutritional status. The MNA-SF has been used to classify subjects as "well-nourished" (score 12-14), "at risk for malnutrition" (score 8-11) or "malnourished" (score 0-7) [21].

To improve the treatment and prognosis, it would be useful to simultaneously conduct a nutritional assessment and evaluate the mental status of elderly patients early in the admission. Therefore, using the STMT-R and MNA-SF, we examined the age, gender and clinical outcomes of geriatric patients admitted to the emergency ward. We aimed to appraise the impact of both cognitive impairment and the nutritional state on mortality and the length of hospitalization among acutely ill geriatric patients and examine the correlation between them.

\section{Subjects And Methods}

Study design

We conducted a cross-sectional, observational study in the ED and acute care ward of Yamamoto Memorial Hospital.

\section{Participants}

Yamamoto Memorial Hospital is an emergency medical institution with a rehabilitation wing located in Imari City, which has a population of about 60,000 . We conducted a survey of non-critically ill patients aged over 50 years old admitted to the emergency department and acute care ward between 1. December, 2014. and 30. September, 2015. Eligible patients were approached during a period of symptom stabilization (when conversation was possible) within a week (almost 2 days) after admission. Screening for cognitive impairment, dementia, and delirium is conducted routinely during hospital admissions [17]. However, to enroll patients in our study, we sought verbal consent from participants or their families in order to ensure the appropriateness of conducting the assessment for research [17]. The findings of all assessments and consents attainment were documented in the medical notes so that they could be freely accessed by clinical teams [17].

Screening assessments and the diagnosis 
STMT-R

We revised the STMT and adapted the instructions for acutely ill elderly patients to provide them with more time and two opportunities to respond to the questionnaire (STMT-R) (Table 1). To assess the feasibility of the STMT-R, it was initially compared with the MMSE in 85 outpatients from our memory impairment clinic. We observed a significant positive correlation between the 2 measures $(r=0.66 ; p<$ 0.001 ) [17], so therefore, the method was adopted to assess cognitive impairment in daily practice.

The diagnosis of cognitive dysfunction

Independently of the first or second attempt to respond to the questions, 1 and 0 points were given for right and wrong answers, respectively [18]. The STMT cut-off value of 4 out of 8 was also used for the STMT-R. Patients with scores $\leq 4$ were therefore classified into the cognitive dysfunction group (CDG), while those with scores $\geq 5$ were classified into the non-cognitive dysfunction group (NCDG) [17]. The test is normally completed within 2 minutes, but some participants were unable to complete the questionnaire within 5 minutes (probably due to severe dementia, delirium, dementia with auditory disturbances, etc.) [17]. Therefore, even if patients had an inspection value of 0 , they were classified into the incomplete test group (ITG) with advanced cognitive impairment from our previous study [17].

\section{MNA-SF}

The MNA-SF is used by trained health professionals and nutritionists for both screening and evaluating the nutritional status [22]. In the present study, the MNA-SF was used to classify subjects into a "wellnourished" group (score 12-14; WNG), an "at risk for malnutrition" group (score 8-11; ARMG) or a "malnourished" group (score 0-7; MNG).

Sample and procedure

Using the STMT-R and NMA-SF, we conducted a nutritional assessment and evaluated the mental status of elderly patients simultaneously at early admission and examined the age, gender and clinical outcomes of the geriatric patients admitted to the ED and acute care ward. The follow-up data on death were collected until September 30, 2019. The date of death was obtained from direct letters, hospital and nursing home clinical notes and social worker networks.

Statistical analyses

The software programs BellCurve for Excel, ver. 2.0 (Social Survey Research Information Co., Ltd., Tokyo, Japan) and JMP® 12 (SAS Institute Inc., Cary, NC, USA) were used for the analyses, with the significance level set at $p<0.05$. We used unpaired t-tests, a chi-square test and an analysis of variance (ANOVA) for univariate analyses. Cox's proportional hazards regression models were then used to calculate the hazard ratios (HRs) and 95\% confidence intervals (Cls). Survival rates were estimated using Kaplan-Meier method. 


\section{Results}

Participants' characteristics

Between December 2014 and September 2015, 976 patients were approached, 249 of whom were excluded for certain reasons (those admitted with critical diseases, receiving sedative medication, unable to consent, or who refused to participate, and those with more than one week of hospitalization). There were 727 participants who met the inclusion criteria and were enrolled in the study $(52.7 \%$ female, mean age: 78.9 years old). Among these, 118 were unable to complete the test (ITG). Among 609 patients who completed the test, 385 had cognitive dysfunction (CDG), and 224 did not have cognitive dysfunction (NCDG). Based on the MNA-SF measured simultaneously, 370, 299 and 58 patients were assigned to the MNG, ARNG and WNG, respectively (Fig. 1).

The majority of hospitalized patients were in their $80 \mathrm{~s}$, but there was no significant difference in age between genders. When the STMT-R scores and age were compared, we noted that there were more patients in the CDG and ITG as age increased, which suggests that the cognitive function declines with age. Regarding the nutritional state, we also observed that there were more patients in the MNG and ARNG as age increased, which suggests that the nutritional state declines with age.

Influence of Underlying Disease

Participants had been admitted for the treatment of several underlying diseases, including musculoskeletal diseases $(n=115,15.8 \%)$, surgical diseases $(n=35,4.81 \%)$, gastroenterological diseases $(n=147,20.2 \%)$, circulatory diseases $(n=151,20.8 \%)$, neurologic diseases $(n=73,10.0 \%)$, metabolic diseases (diabetes, CKD, etc.; $n=108,14.9 \%$ ) and respiratory diseases (almost pneumonia; $n=98,13.5 \%$ ). When comparing rates of diseases among groups classified according to the STMT-R and MNA-SF scores, the percentage of ITG and MNG (41.8\% and 79.6\%) in the respiratory disease group was higher than in the other disease groups (Fig. 2). Underlying respiratory disease may therefore influence the cognitive function and nutritional decline.

Relationships between the cognitive function and nutritional state and the duration of hospitalization

We compared the average hospital stay among the groups. We observed the longest hospitalizations in the ITG ( $66.3 \pm 4.48$ days) and MNG ( $55.5 \pm 2.52$ days) followed by the CDG ( $43.4 \pm 2.48$ days) and ARNG (34.7 \pm 2.78 days), and then the NCDG ( $34.9 \pm 3.24$ days) and WNG ( $24.8 \pm 6.32$ days), with significant differences noted. Furthermore, when comparing the hospital stay by STMT-R and NMA-SF scores, we observed a significant difference between the CDG and ITG for NCDG, and the MNG and ARNG for WNG (Student's t-test), which suggests that the hospital stay becomes longer with the decline in the cognitive function and nutritional state (Fig. 3).

Influence of the cognitive function and nutritional state on the hospital outcome 
We also compared the final hospital outcome for enrolled patients (discharged to nursing home, discharged home, died, or hospital transfer) among the STMT-R and MNA-SF groups. In the STMT-R groups, we observed higher respective rates of discharge to nursing homes and hospital death respectively in the CDG $(17.2 \%$ and $12.8 \%)$ and the ITG $(40.7 \%$ and $39.0 \%)$ than in the NCDG $(7.14 \%$ and $5.36 \%)(p<0.001$ for all; chi-square test). In addition, in the MNA-SF groups, we observed higher respective rates of discharge to nursing homes and hospital death respectively in the ARNG $(9.03 \%$ and $2.68 \%)$ and MNG (27.7\% and $26.9 \%)$ than in the WNG (1.61\% and $1.61 \%)(p<0.001$ for all; chi-square test). Furthermore, the rates of discharge to home in ITG (14.4\%) and MNG (38.0\%) were lower than in the NCDG $(79.5 \%)$ and WNG $(94.8 \%)$. The in-hospital death rate $(n=107)$ was predominant in cognitive functional decline group of ITG $(n=46,43.0 \%)$ and CDG $(n=49,45.8 \%)$, but MNG $(n=99,92.5 \%)$ was predominantly high in the MNA-SF groups (Fig. 4). Naturally, it was found that cognitive decline and poor nutritional status at admission lead to poor hospitalization prognosis.

Relationship between the nutritional status and cognitive decline using the STMT-R

Several studies have shown that the nutritional status is associated with impaired cognition in aging adults in a variety of settings, ranging from the hospital to living in the community [22]. However, the cross-sectional design of those studies has prevented any conclusions from being drawn concerning whether malnutrition causes cognitive decline or vice versa [4]. We examined the relationship between the nutritional status and cognitive function using the STMT-R. We observed a significant positive correlation between STMT-R scores and NMA-SF points $(r=0.523 n=727 ; p<0.001)$, and the nutritional status group in ITG (118 patients; severe cognitive decline group) was predominantly MNG (85.4\%) (Fig. 5). As the cognitive function gets worsens, the proportion of malnutrition increases. This suggests that there is a complex relationship between cognitive impairment and the nutritional status $[4,6]$.

The cognitive function, nutritional state and mortality

Cox's proportional-hazards regression models were used to examine the relationship between the cognitive impairment and malnutrition during follow-up, after adjustment for age (in one-year increments) and gender. In supplementary analyses, we also adjusted for infection (pneumonia and UTI) and cancer treatments at admission. Subjects were followed until September 30, 2019. We analyzed the hazard ratios for mortality (Table 2). Significant HRs were observed for age (per 1-year-of age; HR: $1.060,95 \% \mathrm{Cl}$ : 1.044-1.077, $p<0.0001$ ), male gender (HR: 1.563, 1.214-2.012, $p<0.001$ ), cancer patient (HR: 2.429, 1.733-3.404, $p<0.0001$ ), ARNG (HR: 2.447, 1.010-8.057, $p<0.05$ ), MNG (HR: 4.222, 1.756-13.86, $p<$ 0.001), CDG (HR: 1.844, 1.283-2.718, p<0.001)and ITG (HR: 3.883, 2.567-5.980, p<0.0001). Mild to moderate cognitive impairment(CDG)and ARNG was also found to affect mortality.

Effects of the cognitive function and nutritional status on survival rates

Since the relationship between the cognitive function and nutritional status is complicated, the KaplanMeier method was used to measure cumulative survival according to combinations of the cognitive functional status and the nutritional status during the 4-5-year follow-up period until September 
2019.The survival rate of the combination of ITG and MNG was the worst at $23.58 \%$, while that of CDG and MNG was $51.00 \%$ (Table 3). As the cognitive function and nutritional status declined, so did the survival rate. However, elderly acute patients with a moderate or higher cognitive function may have improved survival rates, even if they are malnourished.

\section{Discussion}

Elderly people tend to have various nutritional problems, such as difficulty swallowing, chewing difficulty and ultimately issues of malnutrition $[19,23]$. If the food intake is low, the threshold of nutrients necessary for a normal brain function will also be low [19]. Several geriatric syndromes, including cognitive disorders, are considered to be a general disorder state in the elderly population [2]. In the elderly, functional deterioration may be the result of cognitive impairment [24]. Increases in mortality and dysfunction are related to the generally poor nutritional status of the elderly [24]. Infections, vulnerability disorders and poisoning are common, and complications of weight loss and malnutrition leading to general health condition changes, cognitive disorders and other geriatric syndromes are attributed to malnutrition in the elderly [19]. Recent studies have shown that the severity of cognitive impairment is positively correlated with weight loss, recurrent infection and frequent hospitalization [25]. Nutritional intervention has been concluded to be beneficial for high-risk groups, and the nutritional assessment of patients with dementia is of critical importance [26].

The relationship between the nutritional status of the elderly and the cognitive function is well documented and seems strong $[4,6]$. However, the cross-sectional design of previous studies has prevented any conclusions from being drawn concerning whether malnutrition causes cognitive decline or vice versa [4]. The relationship between the nutritional status and cognitive performance is complex [6]. The presence or risk of malnutrition may affect the cognitive ability [26], and the presence of cognitive decline affects the ability to perform daily living activities [27] and consume food, which therefore may increase nutritional risks $[27,28]$. In the present study, we aimed to appraise the impact of both cognitive impairment and the nutritional state on hospital outcomes and mortality in acutely ill geriatric patients and to examine the correlation between the cognitive function and nutritional state. We also considered the importance of nutrition management in elderly people with cognitive impairment.

In our study, a positive correlation was found between the STMT-R and MNA-SF scores measured simultaneously in acutely ill geriatric patients $(r=0.5227 n=727 ; p<0.001)$, and cognitive dysfunction was thought to be correlated with malnutrition. This result suggests that the cognitive function may be improved by nutritional intervention and comprehensive rehabilitation.

A recent meta-analysis demonstrated the long-term benefits of oral nutritional supplements (ONS) for cognition, as shown by an improved MMSE score, in dementia patients $[4,29,30]$. However, in severe dementia patients with symptoms of MNG and ARNG, such as those with ITG, a poor prognosis (discharging to the elderly home or death) was also observed with normal nutritional therapy, including ONS. Advanced dementia is associated with feeding problems, including difficulty swallowing and 
respiratory diseases [31]. Whether or not interventions to improve the nutritional status have a positive impact on the cognitive performance, depending on the severity, is unclear [32]. However, Volkert et al. reported that nutrition interventions in the early stages of hospitalization are effective in improving the prognosis of the patients with mild to moderate cognitive impairment where undernourishment is relatively reversible but not in patients with severe dementia or in the terminal phase of life [32]. Our study also suggests that a moderate or higher cognitive function may be associated with improved survival, even with malnutrition. Cognitive decline was thought to affect mortality more than malnutrition.

With regard to predicting the prognosis and outcome according to the STMT-R and MNA-SF scores, we noted that as the cognitive function and nutritional state declined, the duration of hospitalization tended to be prolonged, along with a reduction in the rate of patients returning home, an increase in the rate of patients entering elderly care facilities and an increase in the mortality rate. In addition, regarding the nutritional status at admission, the majority of hospitalized patients were classified into the MNG, and the ratio of this group among cases of hospital death was particularly high. While nutrition intervention was performed in this group, the cognitive dysfunction and nutritional decline were considered to have adversely affected the hospital outcome and long-term survival. Thus, both the STMT-R and MNA-SF, which can be measured within 5 minutes, are simple and sensitive evaluations for acutely ill elderly patients. A combined survival assessment with both early simultaneous measures is expected to be useful for predicting the effects of comprehensive nutritional interventions, and hospitalization and the post-discharge prognosis in acute elderly patients with cognitive decline.

\section{Limitations}

Several limitations associated with the present study warrant mention. First, the subjects also considered cases of senile dementia, so we surveyed hospitalized patients over 50 years of age. Secondly, the lengthy of hospitalization for enrolled patients was due to the age of the community-dwelling patients and the tendency to have rehabilitation functions in the Japanese acute care hospitals in the county, not including acute patients who were discharged within a week. There is thus some degree of cultural bias in the background. Third, in most cases, the tests were performed on the second day after admission, but in some cases, the tests were performed at an appropriate time within one week due to patient fatigue, clinical disruption, or a generally destructive ED environment [33]. Similarly, the examination time was limited. In future trials, the suitability of this test as a simple screening tool or alternative can be evaluated, but we want to improve the accuracy so that we can select the most suitable and available instrument for ED applications as an indicator of medical intervention [33]. Finally, our study suggests that early nutritional interventions may improve the prognosis for patients with a good cognitive function [4]. For clinicians, recognition of cognitive impairment and malnutrition promotes disposal decisions early in hospitalization [34], ensures clarity of discharge instructions [33, 35]. Future trials of ED-based cognitive dysfunction and malnutrition case-finding will need to assess the effects of such screening on patient-oriented outcomes, like preventable recidivism (including readmission), functional decline, and the quality of life of patients and caregivers after discharge [33]. 


\section{Conclusion}

In our research, cognitive impairment and malnutrition at admission had a negative effect on mortality and the hospital outcomes in acutely ill geriatric patients and were closely related to one another. Thus, increased awareness of ED clinicians and medical staffs about possible cognitive impairment and malnutrition facilitates the early initiation of interventions (nutrient management, rehabilitation, etc.) to improve the functional independence and prognosis [33].

\section{Availability Of Data And Materials}

The datasets used and/or analysed during the current study are available from the corresponding author on reasonable request.

\section{Abbreviations}

STMT-R

Revised simplified short-term memory recall test

MNA-SF

Short-form mini-nutritional assessment

ITG

Incomplete testing group

CDG

Cognitive dysfunction group

NCDG

Non-cognitive dysfunction group

MNG

"malnourished" group

ARNG

"at risk of malnutrition" group

WNG

"well-nourished" group

ED

Emergency department

MMSE

Mini-Mental State Examination

HR

Hazard ratio

$\mathrm{Cl}$

Confidence interval

A/D

Alive/Dead 
CSR

Cumulative survival rate

SE

Standard error

\section{Declarations}

\section{Acknowledgments}

Authors wish to thank study participants, data collectors, and study hospital administrators who contributed to this study.

\section{Funding}

There are no sources of funding.

\section{Author information}

Affiliations

Department of Acute Care Medicine in Yamamoto Memorial Hospital

88-4 Hachiyagarami, Niri-cho, Imari, 848-0031 Japan

Hiroshi Yamamoto and Yasushi Hisamatsu

Department of Internal Medicine in Yamamoto Memorial Hospital

Kenichi Ogawa and Toshifumi Morooka

Department of Environmental Medicine, Kurume University School of Medicine, 67 Asahi-machi, Kurume 830-0011 Japan

Tatsuya Ishitake

\section{Contributions}

Conception and design of the research: HY, KO; acquisition of data: HY, KO, TM, YH; analysis and interpretation of the data: $\mathrm{HY}, \mathrm{KO}$ statistical analysis: $\mathrm{HY}, \mathrm{Tl}$; drafting of the manuscript: $\mathrm{HY}, \mathrm{KO}, \mathrm{Tl}$; critical revision of the manuscript for important intellectual content: $\mathrm{HY}, \mathrm{KO}, \mathrm{TI}$. All authors read and approved the final manuscript.

\section{Corresponding author}

Correspondence to Hiroshi Yamamoto 


\section{Compliance with Ethical Standards}

\section{Conflict of Interest}

The authors declare that they have no conflict of interest.

\section{Ethical Approval and Consent to Participate}

All procedures performed in studies involving human participants were in accordance with the ethical standards of the institutional and/or national research committee and with the 1964 Helsinki Declaration and its later amendments or comparable ethical standards. Informed consent was obtained from all individual participants included in the study. All procedures were approved by the ethical review board of the Yamamoto Memorial Hospital.

\section{Consent for Publication}

The manuscript has been approved by all the authors. All authors concur with the submission.

\section{References}

1. Carpenter C. R., DesPain B. , Keeling T. N., Shah M. and Rothenberger M. The SixItem Screener and AD8 for the Detection of Cognitive Impairment in Geriatric Emergency Department Patients Ann Emerg Med. 2011 57: 653-661.

2. Inouye SK, Studenski S, Tinetti ME, et al. Geriatric syndromes clinical, research, and policy implications of a core geriatric concept. J Am Geratr Soc. 2007; 55:780-791

3. Sauvaget C, Yamada M, Fujiwara S, Sasaki H Dementia as a Predictor of Functional Disability: A Four-Year Follow-Up Study: Gerontology 2002;48:226-233 
4. Alish C.: The Connection between Malnutrition and Cognitive Decline in Older Adults: Abbott Nutrition Health Institute Science. Institute

5.Saka B, Kaya O, Ozturk GB, Erten N, Karan MA Malnutrition in the elderly and its relationship with other geriatric syndromes: Clinical nutrition, 2010 454-462. 16

6. Fagerström $\mathrm{C}^{1}$, Palmqvist R, Carlsson J, Hellström Y. Malnutrition and cognitive impairment among people 60 years of age and above living in regular housing and in special housing in Sweden: a population-based cohort study. Int J Nurs Stud. 2011 Jul;48(7):863-71

7.Ministry of Internal Affairs and Communication, Statistics Bureau. "Japan Statistical Yearbook, Chapter 2: Population and Households". Retrieved 13 January 2016.

8.Cabinet Office, Government of Japan, Statistics Bureau. Available at: http://www8.cao.go.jp/kourei/whitepaper/w-2017/html/gaiyou/s1_2_3.html (in Japanese)

9.Japanese Nursing Association, "Nursing for the older people in Japan" Available at: https://www.nurse.or.jp/jna/english/pdf/info-02.pdf

10.LaMantia M, Stump T, Messina F, Miller D, Callahan C; Emergency Department Use Among Older Adults with Dementia Alzheimer Dis Assoc Disord. 2016 Jan-Mar; 30(1): 3540.

11. George J, Long S, Vincent C.: How can we keep patients with dementia safe in our acute hospitals? A review of challenges and solutions. J R Soc Med. 2013 Sep;106(9):355-61.

12. Wilber ST, Carpenter CR, Hustey FM. The Six-Item Screener to detect cognitive impairment in older emergency department patients. Acad. Emerg. Med. 2008; 15:613-616.

13. Guigoz Y. The Mini-Nutritional Assessment (MNA $\left.{ }^{\circledR}\right)$ Review of the Literature - What does it tell us? J Nutr Health Aging 2006; 10:466-487.

14. Allen VJ, Methven L, Gosney MA.: Use of nutritional complete supplements in older adults with dementia: systematic review and meta-analysis of clinical outcomes. Clin 
Nutr. 2013 Dec;32(6):950-7.

15.Orsitto G, Fulvio F, Tria D, Turi V, Venezia A, Manca C. Nutritional status in hospitalized elderly patients with mild cognitive impairment. Clin Nutr. 2/2009 2009;28(1):100-102.

16. Orlandoni P., Claudia Venturini, Nikolina Jukic Peladic, Annarita Costantini, Mirko Di Rosa: Malnutrition upon Hospital Admission in Geriatric Patients: Why Assess It?

Front Nutr. 2017; 4: 50. Published online 2017 Oct 30

17. Yamamoto H., Ogawa K., Battifora H.H., Yamamuro K and Ishitake T.: Assessment and Clinical Implications of Cognitive Impairment in Acutely Ill Geriatric Patients using a Revised Simplified Short-Term Memory Recall Test (STMT-R) Aging Clin Exp Res, March 2019, Volume 31, Issue 3, pp 345-351

18. Kobayashi N., Nakano K., Tago H. and Niwa S.: Development of a simplified ShortTerm Memory recall Test (STMT) and its clinical evaluation, Aging Clin Exp Res, 2010; 22: 157-163.

19. Daradkeh G. , Essa M.M., Al-Adawi S.S., Koshy R.P., Al-Asmi A.A., Waly M.I.: Nutritional Status and Cognitive Impairment in Elderly Pakistan Journal of Biological Sciences 2014 Vol.17(10): 1098 - 1105

20. Vellas B, Villars H, Abellan G, et al. Overview of the MNA ${ }^{\circledR}$ - Its History and Challenges. J Nutr Health Aging 2006; 10:456-465.

21. Rubenstein LZ, Harker JO, Salva A, Guigoz Y, Velas B. Screening for Undernutrition in Geriatric Practice: Developing the Short-Form Mini Nutritional Assessment (MNA-SF). J. Geront 2001;56A: M366-377.

22. Wells JL, Dumbrell AC: Assessment and Treatment of Compromised Nutritional Status in Frail Elderly Patients Clin. Interv. Aging 2006 Mar; 1(1): 67-79.

23. Hickson M.: Malnutrition and ageing Postgrad Med J. 2006 Jan; 82(963): 2-8 
24. Auyeung TW, Kwok T, Lee J, Leung PC, Leung J, Woo J.: Functional decline in cognitive impairment--the relationship between physical and cognitive function Neuroepidemiology. 2008;31(3):167-73.

25. Ahmed T, Haboubi N.: Assessment and management of nutrition in older people and its importance to health. Clin Interv Aging. 2010 Aug 9;5:207-16

26. Malara A., Sgrò G., Caruso C. et al: Relationship between cognitive impairment and nutritional assessment on functional status in Calabrian long-term-care Clin Interv Aging. 2014; 9: 105-110.

27. Sands LP, Yaffe K, Lui LY, Stewart A, Eng C, Covinsky K.: The effects of acute illness on ADL decline over 1 year in frail older adults with and without cognitive impairment. J Gerontol A Biol Sci Med Sci. 2002; 57: M449-54.

28. Martin C.R. and Preedy V. R.: Diet and Nutrition in Dementia and Cognitive Decline Book 2015

29. Engelhart S. and Brummer R.: Assessment of nutritional status in the elderly: a proposed function-driven model Mon, 16 Apr 2018 in Food \& Nutrition Research

30. Allen VJ, Methven L, Gosney MA. Use of nutritional complete supplements in older adults with dementia: Systematic review and meta-analysis of clinical outcomes. Clin Nutr. Dec 2013;32(6):950-957.

31. Pivi G.A., Bertolucci P.H., Schultz R.R.: Nutrition in Severe Dementia

Curr Gerontol Geriatr Res. 2012; 2012:983056.

32. Volkert D, Chourdakis M, Faxen-Irving G, Frühwald T, Landi F, Suominen MH,

Vandewoude M, Wirth R, Schneider SM.: ESPEN guidelines on nutrition in dementia.

Clin Nutr. 2015 Dec;34(6):1052-73 
33. Carpenter CR, Bassett ER, Fischer GM, Shirshekan J, Galvin JE, Morris JC.: Four sensitive screening tools to detect cognitive dysfunction in geriatric emergency department patients: brief Alzheimer's Screen, Short Blessed Test, Ottawa 3DY, and the caregivercompleted AD8. Acad Emerg Med. 2011 Apr;18(4):374-84

34. Hustey FM, Meldon SW, Smith MD, Lex CK. The effect of mental status screening on the care of elderly emergency department patients. Ann Emerg Med. 2003;41(5):678-84.

35. Edwards DF, Baum CM, Meisel M, et al. Home-based multidisciplinary diagnosis and treatment of inner city elders with dementia. Gerontologist. 1999;39(4):483-8.

\section{Tables}

Due to technical limitations, Tables 1, 2 and 3 are only available as a download in the supplemental files section.

\section{Figures}




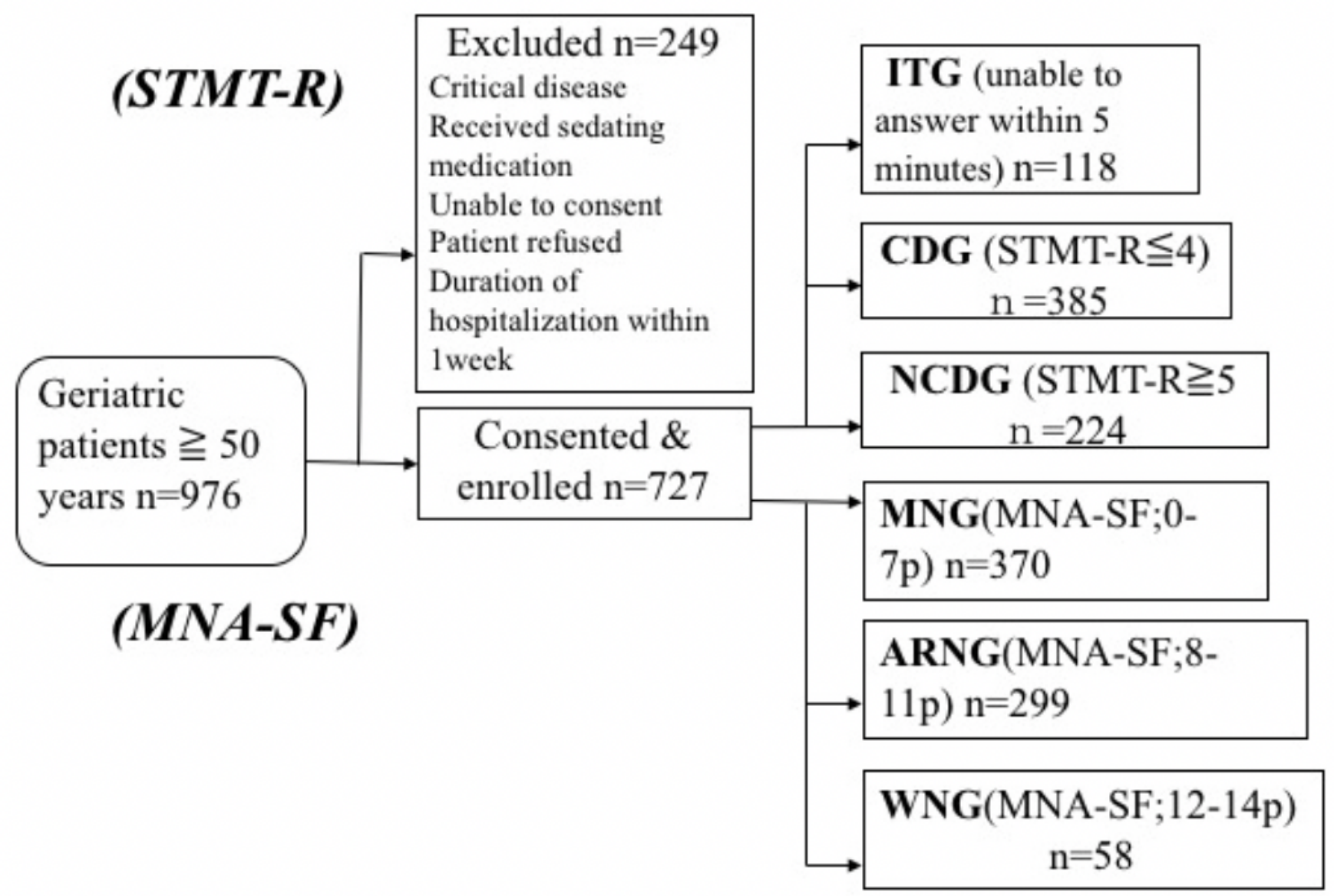

Figure 1

Flow diagram for patient enrollment. NCDG: non-cognitive dysfunction group. WNG: "well-nourished" group. CDG: cognitive dysfunction group. ARMG: "at risk of malnutrition" Group. ITG: incomplete testing group. MNG: "malnourished" group 


\section{Underlying Disease NCDG CDG ITG IVNG ARNG MNG}

Musculoskeletal

Surgical

Gastroenterology

Circulatory

Neurologic

Metabolic

Respiratory
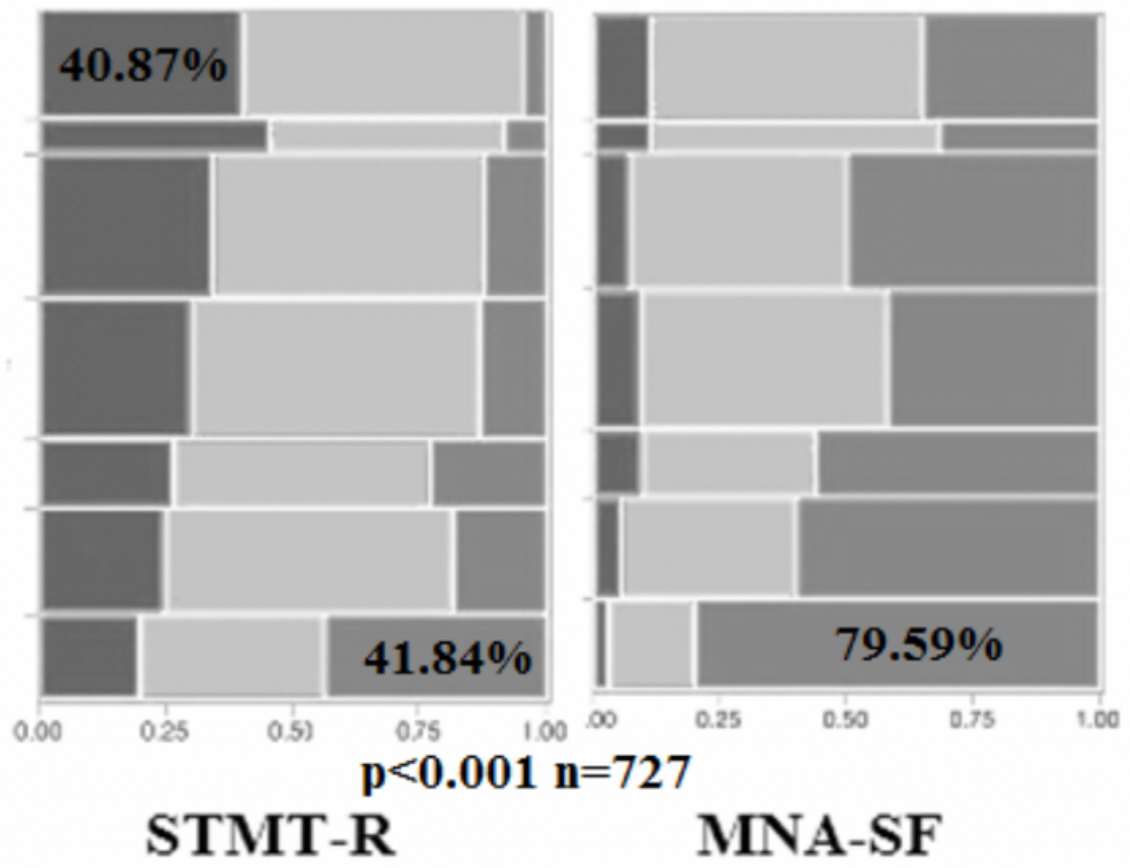

MNA-SF

Figure 2

Proportions of groups by underlying disease ( $\mathrm{p}<0.001$, chi-squared test). The percentage of ITG and MNG $(41.8 \%$ and $79.6 \%)$ in the respiratory disease group was higher than in the other disease groups.

Underlying respiratory disease has been found to reduce the cognitive function and nutritional status.
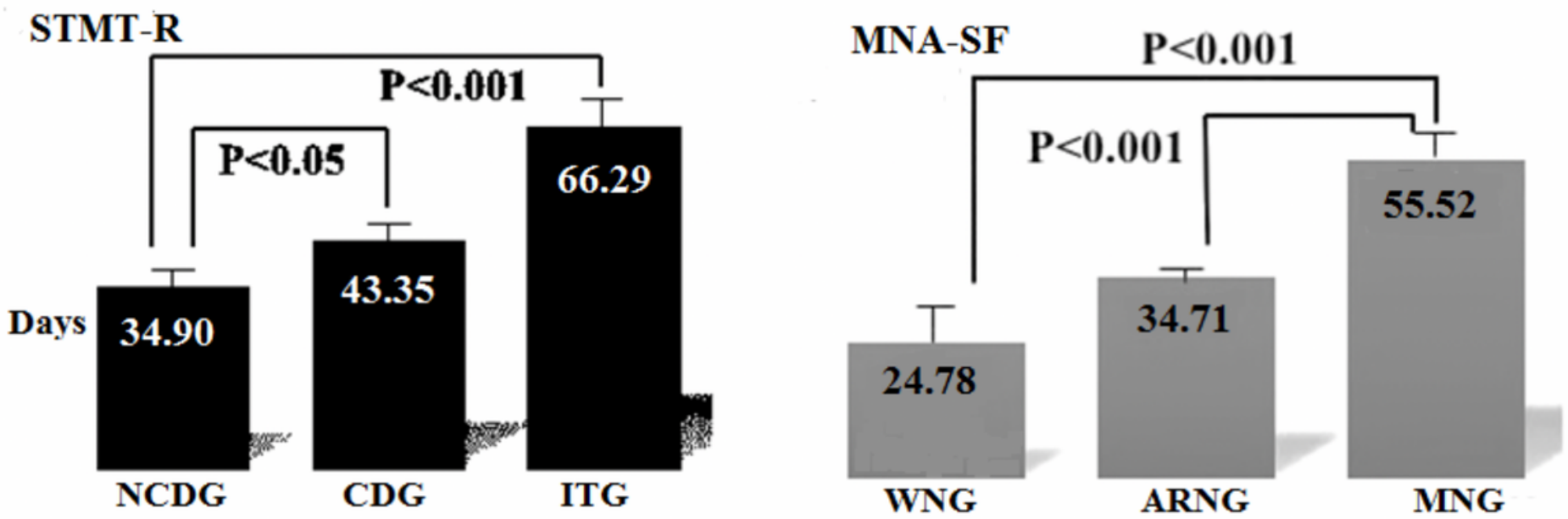

Figure 3 
The comparison of the hospital stays (days) among the STMT-R and MNA-SF groups $(n=727)$. The vertical bars show the mean values and the standard error (SE). Significant differences were found by an ANOVA with multiple comparison.
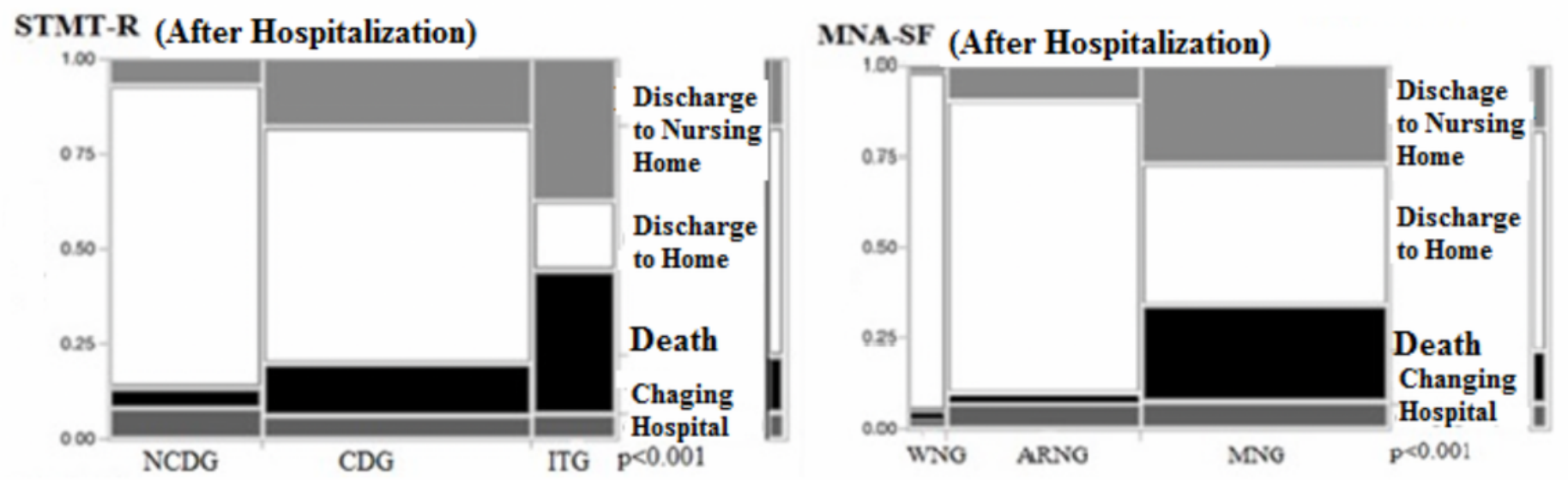

\section{Figure 4}

The hospital outcome by the cognitive function and nutritional status $(n=727)$. Being discharged home may become more difficult as the cognitive function and nutritional status decline $(p<0.001$, chi-squared test).
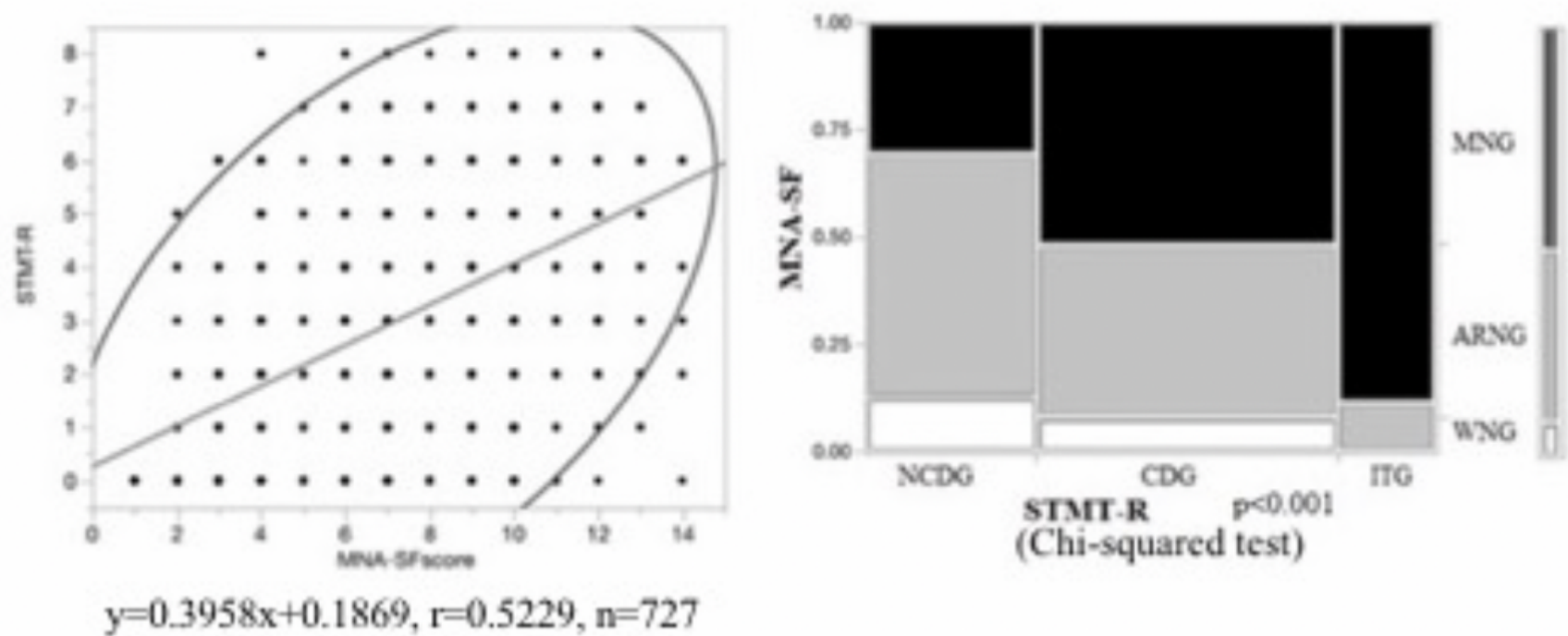

Figure 5

The relationship between the nutritional status and cognitive decline. A significant positive correlation between the STMT-R scores and NMA-SF points $(r=0.5229 n=727 ; p<0.001)$ was observed, and the 
dominant nutritional status in ITG (118 patients; severe cognitive decline group) was by MNG (88.5\%). As the cognitive function worsens, the proportion of malnutrition increases.

\section{Supplementary Files}

This is a list of supplementary files associated with this preprint. Click to download.

- Table2.png

- Table1.png

- Table3.png 